\title{
Confronting Implicit Bias Toward Patients: A Scoping Review of Post-Graduate Physician Curricula
}

Sonya Gleicher ( $\square$ Gleicher@uw.edu )

University of Washington

Morgen Chalmiers

University of California, San Diego

Beverly Aiyanyor

Boston Children's Hospital

Rohini Jain

University of California, San Francisco

Nikhil Kotha

University of California, San Diego

Kristan Scott

Boston University

Robert Sangmok Song

Emory University

Jennifer Tram

University of California, San Diego

Caresse Vuong

Advocate Christ Medical Center

Jennifer Kesselheim

Harvard University

\section{Research Article}

Keywords:

Posted Date: February 18th, 2022

DOI: https://doi.org/10.21203/rs.3.rs-1318722/v1

License: (1) (1) This work is licensed under a Creative Commons Attribution 4.0 International License.

Read Full License 


\section{Abstract \\ Background}

Physicians' behavior may unknowingly be impacted by prejudice and thereby contribute to healthcare inequities, so it is imperative that physicians learn to recognize and minimize implicit bias. Despite increasingly robust data demonstrating physician implicit bias, ${ }^{1,2}$ the evidence behind how to change this with training programs remains unclear. This scoping review therefore reports on the implementation, outcomes, and characteristics of post-graduate physician implicit bias curricula.

\section{Methods}

The authors conducted a literature review using scoping review methodology. They searched 7 databases in February and November 2020 for English-language academic and gray literature on implicit bias curricula for physicians at all levels of post-graduate training. Ten reviewers screened studies for eligibility independently, then extracted data from these studies and compiled it into a chart and analytical summary.

\section{Results}

Of the 4,599 articles screened, this review identified 90 articles on implicit bias interventions for postgraduate physicians. Inductive data analysis revealed a spectrum of educational approaches, which were categorized into 4 educational models called Competence, Skills-Based, Social Contact, and Critical Models. The most commonly reported strength was the interactive nature of the curricula (26\%), and the most frequently identified challenges were related to time and resources available (53\%). Half of the interventions discussed facilitator preparation, and the majority (62\%) evaluated outcomes using pre and post self-assessments.

\section{Conclusions}

Medical educational interventions should be based on robust data and theory. This review provides a comprehensive synthesis of the literature on physician implicit bias curricula, which can support medical educators by serving as a platform for data, recommendations, and examples of implicit bias training. With more effective and evidence-based implicit bias programs, physicians can help diminish healthcare inequities.

\section{Background}

Longstanding health inequities based on race, gender, socioeconomic status, and other social influencers of health have been the subject of renewed attention in light of current events such as the COVID-19 
pandemic and our national reckoning with systemic racism. ${ }^{1,2}$ The Agency for Healthcare Research and Quality reports that patients of Black, LatinX, or indigenous race receive worse care in relation to $40 \%$ of quality measures assessed, and the annual National Healthcare Disparities Report consistently demonstrates that white patients receive better quality of care than other racial groups. ${ }^{3}$ This differential in care remains after controlling for economic status, educational level, and healthcare access, suggesting discrimination on the part of the medical system, ${ }^{4}$ where covert prejudice remains present at the individual and institutional levels.

Mounting research suggests that implicit bias toward patients has measurable impacts on healthcare inequities. ${ }^{5}$ Implicit bias is an unconscious and unintentional association between a category of people and some attribute. ${ }^{6}$ While explicit attitudes are deliberate and conscious, implicit attitudes can affect behavior without conscious volition. ${ }^{3}$

Physicians require evidence-based training in order to combat these preformed schemata, yet the availability of opportunities for physicians to explore their biases in a formal setting after medical school is unclear. It is critical to involve post-graduate physicians because they are responsible for making clinical decisions and training future generations of physicians, yet no literature review has been conducted on post-graduate physician implicit bias interventions. ${ }^{7-9}$

We conducted a comprehensive scoping review to present the content, outcomes, and recommendations of educational interventions which address post-graduate physicians' implicit bias toward patients, such that a broad audience of medical educators can design similar interventions based on evidence and feasibility.

\section{Methods}

We employed a rigorous scoping review methodology, using the JBI Manual for Evidence Synthesis framework. ${ }^{10}$ Using this strategy, our scoping review was split into the stages below:

\section{Developing the research question}

We asked, how can implicit bias toward patients be addressed through physician educational programs?

\section{Inclusion criteria}

The population of focus was post-graduate physicians, such as resident physicians, fellows, and attending physicians of all specialties, including populations in which physicians were a subgroup of a larger group of learners. We focused on curricula addressing implicit bias toward patients and defined implicit bias as stigma, prejudice, stereotype, and other forms of unconscious bias based on race, socioeconomic status, sexual orientation, weight, substance use, and any other personal identifying trait. We defined curricula as any planned educational experiences, including clinical rotations, didactics, training programs, and conferences. Primary research, systematic reviews, books, editorials, guidelines, 
videos, and conference abstracts were included, while non-English language studies were excluded. We included literature describing implemented curricula as well as literature which provided recommendations and theoretical background for potential interventions. We did not limit studies by publication date.

\section{Search strategy}

With the aid of an experienced research librarian (P. Bain, Countway), we conducted a search of MEDLINE (Ovid), Embase, Web of Science, ERIC, CINAHL, and PsycINFO in February 2020 using the search strategy and keywords in Appendix 1. Because we found relevant articles from the database MedEdPORTAL which were not identified in this initial search, we conducted a manual search of MedEdPORTAL in November 2020 using the terms "implicit bias," "unconscious bias," "prejudice," and "stigma."

\section{Sources of evidence selection}

We used Covidence systematic review management software (Melbourne, Australia) for each step of screening and data extraction. First, all reviewers applied inclusion criteria to $10 \%$ of the papers to ensure that we were uniform in our screening. We conducted the remaining screening in two stages: titles and abstracts were screened initially, then the full texts of included articles were screened to determine final eligibility (Fig. 1). All coauthors (S.G., M.C., B.A., R.J., N.K., K.S., R.S., J.T., C.V., and J.K.) participated in both rounds of screening. Each article was independently reviewed by two coauthors using predefined selection criteria and we gleicherresolved disagreements with reviewer discussion until consensus was reached.

\section{Data extraction}

Coauthors (S.G., M.C., B.A., R.J., N.K., K.S., R.S., J.T., C.V., and J.K.) collected data from the included studies using a data extraction form (Appendix 2). The form's data fields were guided by educational principles deemed most relevant by the coauthors as well as the Guideline for Reporting Evidence-Based Practice Education Interventions and Teaching (GREET) checklist. ${ }^{12}$

\section{Analysis of the evidence}

Three coauthors (S.G., M.C., and J.K.) analyzed data qualitatively and quantitatively, using frequency counts for key characteristics identified. Interventions were categorized into 4 distinct educational models developed iteratively via inductive coding by the authors. We analyzed outcomes using Kirkpatrick's 4 levels of program evaluation, an analytic model for curricular outcome measurement. ${ }^{13}$

\section{Results}

\section{Curriculum characteristics}

Our review identified 90 articles on implicit bias interventions for post-graduate physicians. Table 1 presents the aggregated data from these articles, and Appendix $\mathbf{3}$ summarizes characteristics of all 90 
articles.

Page 5/21 
Table 1

Aggregated data from 90 studies included in scoping review on post-graduate physician implicit bias curricula.

\section{Type of bias addressed}

n (\%)

Total

reported: 90

General implicit bias

$41(46 \%)$

Race, ethnicity, and diverse cultures

$21(23 \%)$

LGBQ Patients

$7(8 \%)$

Mental Illness

$6(7 \%)$

Socioeconomic Status

$6(7 \%)$

Other $20(22 \%)$

Including bias related to HIV/AIDS, weight/obesity, gender, substance use disorders, disability, age, gender non-conforming/intersex, and incarcerated populations

\section{Learners' professional position}

n (\%)

Total reported: 82

Residents/fellows $53(65 \%)$

Attendings $26(32 \%)$

Physicians: unspecified $20(24 \%)$

Mixed health professionals $18(22 \%)$

Nurses, social workers, and other members of the health care system

Medical students $13(16 \%)$

Learners' specialty $\mathrm{n}(\%)$

Total reported: 49

Internal medicine $17(35 \%)$

Including general internal medicine, hematology-oncology, endocrinology, and primary care

Family medicine

$9(18 \%)$

Emergency medicine

Pediatrics

Open to multiple specialties 


\section{Psychiatry}

Other
$4(8 \%)$

$5(10 \%)$

Including OB/GYN, physical medicine and rehabilitation, surgery, and palliative care

\section{Curriculum schedule}

$\mathrm{n}(\%)$

Total reported: 52

Single session

6 months or more

1 month to $<6$ months

1 week to $<4$ weeks

2 days to $<7$ days

Mode of intervention

Group discussion, exercise, or debrief

Lecture, didactic, or reading

Exposure to patient population or community members

Reflection exercise or writing

Film

Role play or simulation

IAT

Case-based learning

Asynchronous online module or e-learning

Was facilitator background/preparation reported?
$28(54 \%)$

$11(21 \%)$

8 (15\%)

4 (8\%)

1 (2\%)

n (\%)

Total reported: 73

49 (67\%)

41 (56\%)

$20(27 \%)$

$16(22 \%)$

$15(21 \%)$

$13(18 \%)$

11 (15\%)

10 (14\%)

5 (7\%)

n (\%)

66

implemented curricula

33 (50\%)

$33(50 \%)$

No 


\section{Methods for measuring outcomes}

n (\%)

Total reported: 58

Pre and post surveys $36(62 \%)$

Post surveys/course evaluations $19(33 \%)$

Interviews/focus groups

$8(14 \%)$

Observation of clinical decision-making

$3(5 \%)$

Long-term follow-up surveys

$3(5 \%)$

Other

Includes written reflections and IAT

\section{Outcomes reported}

Increased recognition of systemic disparities

Increased awareness of personal bias

Significant reduction in measured bias

Increased comfort in or commitment to addressing bias

Learners rated intervention highly

Self-reported reduction in discriminatory behavior

Increased knowledge of strategies to address bias

Increased understanding of patients' experiences

Increased insight into teaching about bias

Other:

Includes significant increase in measured bias and no significant change in learner behavior

\section{Strengths reported}


Evidence-based

Research or guidelines formed basis for curriculum

Perspective-taking/fosters empathy

Interdisciplinary contributions to curriculum

Involving patients, community, or other fields

Learning environment conducive to honest discussion

Cultural humility/cross-cultural care

Feasibility

Actionable solutions

Provides tools for providers to use to change clinical practice

Simulated patient encounter

Weaknesses

Lack of time/resources

Includes scheduling challenges, brief duration of intervention, and lack of faculty/institutional investment

Learner defensiveness (including distrust of IAT validity)

Lack of facilitators experienced in/comfortable with subject material

Learners self-selected and may not represent target audience

Lack of actionable solutions

$4(11 \%)$

Limited scope of course material

$3(8 \%)$

Subject undervalued by learners

$3(8 \%)$

Risk of reinforcing stereotypes

$2(6 \%)$ 
Type of bias addressed

\begin{tabular}{lc|} 
& $\begin{array}{c}\text { Total } \\
\text { reported: } 90\end{array}$ \\
\hline Future directions & $\mathrm{n}(\%)$ \\
& $\begin{array}{l}\text { Total } \\
\text { reported: } 45\end{array}$ \\
\hline $\begin{array}{l}\text { Improve outcomes evaluation (including behavioral outcomes and long-term } \\
\text { outcomes) }\end{array}$ & $19(42 \%)$ \\
\hline Extend to more sessions & $7(16 \%)$ \\
\hline Improve facilitator preparation & $4(9 \%)$ \\
\hline Encourage institutional buy-in & $3(7 \%)$ \\
\hline $\begin{array}{l}\text { Interdisciplinary and community collaboration } \\
\text { Includes partnerships with community, patients, and other disciplines }\end{array}$ & $3(7 \%)$ \\
\hline Reevaluate competency model & $3(7 \%)$ \\
\hline Examine alternatives to the cultural competency model for teaching implicit bias & \\
\hline More clinical immersion & $3(7 \%)$ \\
\hline
\end{tabular}

\section{Educational models}

Inductive data analysis revealed 4 educational models used in implicit bias curricula: Competence, SkillsBased, Social Contact, and Critical Models. Their different theoretical foundations and pedagogical approaches are summarized in Table 2. 
Table 2

Educational models identified in curricula addressing post-graduate physicians' implicit bias toward patients.

\begin{tabular}{|c|c|c|}
\hline \multirow{2}{*}{$\begin{array}{l}\text { Educational } \\
\text { model }\end{array}$} & \multirow[t]{2}{*}{ Description } & $\mathrm{n}(\%)$ \\
\hline & & $\begin{array}{l}\text { Total } \\
\text { reported: }\end{array}$ \\
\hline $\begin{array}{l}\text { Competence } \\
\text { Models }\end{array}$ & $\begin{array}{l}\text { Seek to increase learners' knowledge about diverse populations and } \\
\text { awareness of their own implicit bias, often via self-reflection exercises. } \\
\text { Often informed by Pedersen's }{ }^{14} \text { foundational } \\
\text { Awareness/Knowledge/Skills prototype for culture-centered counseling. }\end{array}$ & $\begin{array}{l}30 \\
(54 \%)\end{array}$ \\
\hline $\begin{array}{l}\text { Critical } \\
\text { Models }\end{array}$ & $\begin{array}{l}\text { Contextualize implicit bias within larger systems of inequity and seek to } \\
\text { prepare learners to catalyze structural change that extends beyond } \\
\text { individual clinical interactions. }\end{array}$ & $\begin{array}{l}11 \\
(20 \%)\end{array}$ \\
\hline $\begin{array}{l}\text { Skills-Based } \\
\text { Models }\end{array}$ & $\begin{array}{l}\text { Employ self-reflection combined with training in specific, evidence-based } \\
\text { strategies from Social Cognitive Psychology (e.g., individuation, } \\
\text { perspective-taking). }\end{array}$ & $9(17 \%)$ \\
\hline $\begin{array}{l}\text { Social } \\
\text { Contact } \\
\text { Models }\end{array}$ & $\begin{array}{l}\text { Incorporate evidence from Social Cognitive Psychology to facilitate } \\
\text { interactions between clinicians and diverse patients under conditions }{ }^{15} \\
\text { shown to reduce bias. }\end{array}$ & $6(11 \%)$ \\
\hline
\end{tabular}

\section{Outcomes reported}

Eighty percent of the educational interventions reported outcomes. Outcome assessments most frequently relied on learners to self-report the perceived effects of the curriculum through pre and post surveys (62\%). Figure 2 depicts the interventions' approaches to outcome measurement through the lens of Kirkpatrick's model for program evaluation. ${ }^{13}$

\section{Curriculum analysis}

Most of the educational methods employed were interactive (67\%), and this was the most commonly identified curricular strength (26\%). The most common weaknesses identified were related to resource availability, such as schedule and timing, funding, and institutional investment (53\%).

\section{Discussion}

Our review identified several elements and challenges of effective physician implicit bias curricula. Below we highlight a spectrum of educational approaches to these curricula, as well as areas for improvement in implementation and outcome assessment.

\section{Educational models}


The 4 educational models (Table 2) identified in our analysis present various strengths and weaknesses. Competence Models have been critiqued for presenting implicit bias as a problem to be understood and resolved at the level of the individual, ${ }^{15-17}$ often by increasing learners' awareness of their bias. Although evidence does not support the premise that increased awareness alone will allow clinicians to manage their own implicit bias, ${ }^{18,19}$ self-reflection may trigger cognitive dissonance and increase learner motivation to change. In our review, $20 \%$ of interventions identified self-reflection on personal bias as a strength. On the other hand, when Competence Models are used to improve learners' understanding of cultural groups by focusing on categorical traits rather than individuation, they may have the counterproductive effect of actually increasing reliance on stereotypes. ${ }^{20-22}$ It is critical that interventions demonstrate heterogeneity rather than homogeneity within stereotyped groups, a strength which was recognized in $20 \%$ of curricula published in this review.

Skills-Based Models draw upon evidence-based strategies in Social Cognitive Psychology that have been shown to reduce stereotyping outside of healthcare settings. ${ }^{18,23-25}$ These skills may include "perspective-taking," which fosters empathy by asking learners to imagine themselves in a patient's position. Another practice, called individuation, consciously focuses on "specific information about an individual,"18 which may "increase [learners'] capacity to see others as members of a common ingroup" instead of an outgroup. ${ }^{23}$ Such models may also use mindfulness, which encourages "attention to one's own thought processes... and how they affect decisions so that one pays attention to the details of clinical care rather than falling back on habits...such as stereotypes." ${ }^{20}$

Social Contact Models facilitate direct interaction with diverse patients to foster empathy and enhance learners' comfort, confidence, and positive emotions in interactions with people they perceive to be outgroup members. ${ }^{23,24,26,27}$ Evidence suggests that social contact only leads to these positive outcomes in specific conditions, namely, the presence of shared goals and equal status between both parties. ${ }^{20,27}$ Otherwise, such interactions have the potential to strengthen previously held stereotypes. ${ }^{20,27}$ To address this risk, novel approaches incorporate standardized patient encounters with debriefing. ${ }^{20}$ One downside to Social Contact Models is that lessons learned with specific populations may not be easily applied to other contexts, in contrast to Skills-Based Models, which provide tools meant to be universally applicable.

Critical Models seek to profoundly transform the paradigms through which learners think about equity and justice in the medical system. In contrast to other models, which seek to avoid provoking discomfort or defensiveness among learners, ${ }^{16,20}$ Critical Models intentionally present learners with experiences designed to arouse emotions, destabilize assumptions, and trigger cognitive dissonance. According to transformative learning, ${ }^{19,28,29}$ an educational theory which focuses on adult learning, such an exposure to a "disorienting dilemma" ${ }^{30}$ prompts learners to "engage in a process of self-examination," leading to paradigm shift and skill acquisition. ${ }^{31}$

\section{Curriculum implementation}


Each educational model encountered challenges in its implementation. Our review revealed barriers related to institutional investment and culture, availability of experienced facilitators, and learner-related factors.

Institutional attitudes can support or impede learning by impacting the time and funding available for implicit bias programs. ${ }^{29}$ Given the multiple competing demands for medical staff time, ${ }^{32}$ it is unsurprising that over half of interventions held only a single session, despite concern that "the lessons of a onetime workshop...tend to fade as the volume of work increases, and old practices reassert themselves." ${ }^{33}$ When institutional investment is lacking, the burden is carried by a handful of sometimes overtaxed individuals, as one author recalls, "we had momentum. What we didn't have was money...which was a recipe for a lot of talk and no action... it seemed pretty clear I was going to have to find the funding for it myself." ${ }^{34}$ We also observed an uneven distribution of implicit bias programs between various specialties, illustrating how departmental subcultures may affect the accessibility of such trainings.

Another barrier identified was the availability of facilitators who were comfortable and well-versed in the subject matter. ${ }^{20,26,29,33,35,36}$ Only half of the interventions discussed the training of facilitators. A deficiency of experienced facilitators could detract from curriculum feasibility and quality while compounding variability in learner experiences. Facilitators may be wary of teaching implicit bias because of the sensitivity of the subject matter, inadequate preparation and training, or institutional cultures of silence with relation to bias. ${ }^{29}$ Some questioned the evidence behind implicit bias, or felt antagonized when confronted with inequities in their establishment. ${ }^{34}$ In response, several articles investigated best practices for facilitator training and identified this as a crucial area for future research. ${ }^{15,29,37}$

Implicit bias programs were also impacted by factors related to learners. Multiple studies relayed concerns that the voluntary nature of these curricula meant that attendees were "self-selected," ${ }^{38}$ such that the program may have been "preaching to the choir." Interventions can reach a greater array of learners if their institutions value implicit bias training and support learners in making time for it. ${ }^{26}$ Changing institutional culture may also address another learner-related factor: the defensiveness and feelings of shame, fear $^{29}$ or denial ${ }^{39}$ that may be experienced when confronting one's own bias. Although such discomfort can be part of the process, as in the case of Critical Models, ${ }^{30,31}$ too much discomfort can be counterproductive. Educators should provide a supportive environment to intentionally channel learner discomfort into behavioral change. ${ }^{20,31}$

Environments which support vulnerability and are free of criticism enable learners to experience transformative change. ${ }^{16}$ One study suggested that "self-reflection, self-awareness, discovering... of often shameful past experiences of bias-could only be accomplished through...a non-judgmental environment in which everyone feels comfortable expressing their views with little fear of mockery or embarrassment." ${ }^{16}$ It is also crucial to avoid taxing learners who are underrepresented minorities by treating them as token representatives of their group or expecting them to educate other learners. ${ }^{40}$ 
Educators must strive to "create a learning environment that fosters safety, trust, and respect," "vet speakers, content, and materials carefully," and "employ andragogical versus pedagogical methods of learning" which treat learners as active agents in their own learning. ${ }^{41}$ Striking this balance may be especially difficult when power differentials exist between facilitators and trainees, which reinforces the need for robust faculty development. ${ }^{29}$

\section{Outcomes reported}

Program evaluation is an essential component of curriculum development. ${ }^{42,43}$ Seventeen percent of studies in this review labeled evidence supporting interventions as a strength. This suggests that educators are seeking data to guide curricula, yet $20 \%$ of interventions did not report results. Faculty development initiatives should explicitly encourage educators to create a prospective evaluation plan to measure and disseminate outcomes, so that others may benefit from the lessons learned.

Kirkpatrick's model for program evaluation (Fig. 2) is a well-known paradigm to categorize approaches to outcome measurement. The reported outcomes of included publications most commonly mapped to Level 2: Learning, which relates to learners' knowledge, attitudes, or skills, as well as confidence or commitment to change. ${ }^{13}$ Noteworthy shortcomings exist within this subset of data. While optimal measurement at Level 2 would involve an external evaluator, ${ }^{42}$ many studies reported outcomes via selfassessments, raising concerns about their validity. ${ }^{44}$ As an alternative, several authors measured IAT scores, often in a pre/post-intervention format. The advantages of such an approach are the rigor with which IAT instruments are developed and evidence that the IAT has greater predictive validity than other self-report measures, ${ }^{45}$ but some publications question the validity and precision of IAT-based data. ${ }^{46-48}$

Few included studies attempted to measure outcomes at Kirkpatrick Levels 3-4. Level 3 assesses the degree to which learners apply what they learned, and Level 4 assesses targeted outcomes and organizational benefits. ${ }^{13}$ Although measurement at these higher levels is challenging due to the time, money, and methodologic expertise required, ${ }^{49,50}$ investing in such outcome evaluation would provide the most direct evidence of interventions' effects on physician implicit bias and patient care. ${ }^{13}$

\section{Conclusions}

Our analysis of the literature on post-graduate physician implicit bias curricula highlights opportunities for next steps in the field:

1. Educators addressing physician implicit bias can use this review as a guide for selecting the most fitting educational models and teaching modalities.

2. Institutional investment and faculty development are crucial to changing physician implicit bias. This includes fostering organizational culture open to change, selecting leaders who are supportive of such change, investing time and money into implicit bias curricula, and training faculty to serve as facilitators. 
3. Implicit bias curricula should be evidence-based. ${ }^{51,52}$ This requires more widespread program evaluation using well-validated instruments, and especially assessing changes in physician behavior and impacts on patients.

It is our goal that this review serves as a platform for data, recommendations, and examples of implicit bias training, so that medical educators can critically apply and improve aspects of these interventions rather than "reinventing the wheel."

\section{Limitations}

This scoping review presents an extensive yet incomplete snapshot of implicit bias curricula for physicians. It is limited to the databases we searched, although we identified additional papers through the iterative process of screening included studies' bibliographies. In addition, many articles provided only brief information in the form of an abstract. Each stage of screening, data extraction, and coding likely introduced a degree of bias from the reviewers, which we mitigated by having 2 reviewers reach consensus at each step. Finally, per scoping review methodology, we did not consider the quality of the studies we included. This lack of discrimination should be considered when extrapolating results.

\section{Declarations}

Ethical approval: Not applicable

Consent for publication: Not applicable

\section{Availability of data and materials:}

The datasets supporting the conclusions of this article are available in the following repositories:

MEDLINE (Ovid), https://www.wolterskluwer.com/en/solutions/ovid/ovid-medline-901

Embase, https://www.embase.com/

Web of Science, https://www.webofscience.com

ERIC, https://eric.ed.gov

CINAHL, https://www.ebsco.com/products/research-databases/cinahl-database

PsycINFO, https://www.apa.org/pubs/databases/psycinfo

MedEdPORTAL, https://www.mededportal.org

Competing interests: The authors declare that they have no competing interests.

Funding: None 
Authors' contributions: Each coauthor (S.G., M.C., B.A., R.J., N.K., K.S., R.S., J.T., C.V., and J.K.) participated in all stages of screening and data extraction. Three coauthors (S.G., M.C., and J.K.) analyzed the data and wrote the final manuscript, which was then reviewed by all coauthors.

Acknowledgments: The authors wish to thank Dr. Paul Bain from Countway Library for his assistance with the literature search and Dr. John Burkhardt from the University of Michigan for reviewing the manuscript.

\section{References}

1. The Office of Minority Health. Minority Population Profiles. Accessed June 17, 2021. https://minorityhealth.hhs.gov/omh/browse.aspx?lvl=2\&lvlid=26

2. COVID-19 Shines Light on Health Disparities $>$ National Conference of State Legislatures. Accessed June 17, 2021. https://www.ncsl.org/blog/2020/07/30/covid-19-shines-light-on-healthdisparities.aspx

3. Hall WJ, Chapman MV, Lee KM, et al. Implicit Racial/Ethnic Bias Among Health Care Professionals and Its Influence on Health Care Outcomes: A Systematic Review. Am J Public Health. 2015;105(12):e60-e76. doi:10.2105/AJPH.2015.302903

4. Zestcott CA, Blair IV, Stone J. Examining the Presence, Consequences, and Reduction of Implicit Bias in Health Care: A Narrative Review. Group Process Intergroup Relat GPIR. 2016;19(4):528-542. doi:10.1177/1368430216642029

5. Dehon E, Weiss N, Jones J, Faulconer W, Hinton E, Sterling S. A Systematic Review of the Impact of Physician Implicit Racial Bias on Clinical Decision Making. Acad Emerg Med Off J Soc Acad Emerg Med. 2017;24(8):895-904. doi:10.1111/acem.13214

6. Gonzalez CM, Deno ML, Kintzer E, Marantz PR, Lypson ML, McKee MD. Patient perspectives on racial and ethnic implicit bias in clinical encounters: Implications for curriculum development. Patient Educ Couns. 2018;101(9):1669-1675. doi:10.1016/j.pec.2018.05.016

7. Morris M, Cooper RL, Ramesh A, et al. Training to reduce LGBTQ-related bias among medical, nursing, and dental students and providers: a systematic review. BMC Med Educ. 2019;19(1):325. doi:10.1186/s12909-019-1727-3

8. Sukhera J, Wodzinski M, Rehman M, Gonzalez CM. The Implicit Association Test in health professions education: A meta-narrative review. Perspect Med Educ. 2019;8(5):267-275. doi:10.1007/s40037-019-00533-8

9. Brottman MR, Char DM, Hattori RA, Heeb R, Taff SD. Toward Cultural Competency in Health Care: A Scoping Review of the Diversity and Inclusion Education Literature. Acad Med J Assoc Am Med Coll. 2020;95(5):803-813. doi:10.1097/ACM.0000000000002995

10. Peters M, Godfrey C, Mclnerney P, Munn Z, Trico A, Khalil H. Chapter 11: Scoping Reviews. In: Aromataris E, Munn Z, eds. JBI Manual for Evidence Synthesis. JBI; 2020. doi:10.46658/JBIMES-2012 
11. Moher D, Liberati A, Tetzlaff J, Altman DG. Preferred reporting items for systematic reviews and meta-analyses: the PRISMA statement. BMJ. 2009;339:b2535. doi:10.1136/bmj.b2535

12. Phillips AC, Lewis LK, McEvoy MP, et al. Development and validation of the guideline for reporting evidence-based practice educational interventions and teaching (GREET). BMC Med Educ. 2016;16(1):237. doi:10.1186/s12909-016-0759-1

13. Kirkpatrick D, Kirkpatrick J. Evaluating Training Programs. 3rd ed. Berrett-Koehler Publishers; 2006.

14. Position Paper: Cross-Cultural Counseling Competencies - Derald Wing Sue, Joseph E. Bernier, Anna Durran, Lawrence Feinberg, Paul Pedersen, Elsie J. Smith, Ena Vasquez-Nuttall, 1982. Accessed May 19, 2021. https://journals.sagepub.com/doi/10.1177/0011000082102008

15. White-Davis T, Edgoose J, Speights JB, et al. Addressing Racism in Medical Education An Interactive Training Module. Family Medicine. Published 2018. Accessed January 12, 2021. /familymedicine/2018/may/white-davis-2017-0183/

16. Hannah SD, Carpenter-Song E. Patrolling your blind spots: introspection and public catharsis in a medical school faculty development course to reduce unconscious bias in medicine. Cult Med Psychiatry. 2013;37(2):314-339. doi:10.1007/s11013-013-9320-4

17. Metzl JM, Hansen H. Structural competency: theorizing a new medical engagement with stigma and inequality. Soc Sci Med 1982. 2014;103:126-133. doi:10.1016/j.socscimed.2013.06.032

18. Chapman EN, Kaatz A, Carnes M. Physicians and implicit bias: how doctors may unwittingly perpetuate health care disparities. J Gen Intern Med. 2013;28(11):1504-1510. doi:10.1007/s11606013-2441-1

19. Sherman MD, Ricco J, Nelson SC, Nezhad SJ, Prasad S. Implicit Bias Training in a Residency Program: Aiming for Enduring Effects. Fam Med. 2019;51(8):677-681. doi:10.22454/FamMed.2019.947255

20. Teal CR, Gill AC, Green AR, Crandall S. Helping medical learners recognise and manage unconscious bias toward certain patient groups. Med Educ. 2012;46(1):80-88. doi:10.1111/j.13652923.2011.04101.x

21. Paroz S, Bonvin R, Casillas A, et al. Cultural competence education in a simulated clinical environment: A pilot experience. J Gen Intern Med. 2014;29:S513-S514.

22. Razack S. Promoting skill-building in cultural competence: A must for paediatricians who care for socially vulnerable populations. Paediatr Child Health. 2007;12(8):657-659. doi:10.1093/pch/12.8.657

23. Burgess $D$, van Ryn $M$, Dovidio J, Saha S. Reducing racial bias among health care providers: lessons from social-cognitive psychology. J Gen Intern Med. 2007;22(6):882-887. doi:10.1007/s11606-0070160-1

24. Knaak S, Mantler E, Szeto A. Mental illness-related stigma in healthcare: Barriers to access and care and evidence-based solutions. Healthc Manage Forum. 2017;30(2):111-116.

doi:10.1177/0840470416679413

Page $17 / 21$ 
25. Perdomo Joanna, Tolliver Destiny, Hsu Heather, et al. Health Equity Rounds: An Interdisciplinary Case Conference to Address Implicit Bias and Structural Racism for Faculty and Trainees. MedEdPORTAL. 15:10858. doi:10.15766/mep_2374-8265.10858

26. Agrawal S, Capponi P, López J, et al. From Surviving to Advising: A Novel Course Pairing Mental Health and Addictions Service Users as Advisors to Senior Psychiatry Residents. Acad Psychiatry. 2016;40(3):475-480. doi:10.1007/s40596-016-0533-z

27. Sukhera J, Miller K, Scerbo C, Milne A, Lim R, Watling C. Implicit Stigma Recognition and Management for Health Professionals. Acad Psychiatry.:5. doi:10.1007/s40596-019-01133-8

28. Loignon C, Boudreault-Fournier A, Truchon K, Labrousse $Y$, Fortin B. Medical residents reflect on their prejudices toward poverty: a photovoice training project. BMC Med Educ. 2014;14:1050. doi:10.1186/s12909-014-0274-1

29. Gonzalez CM, Garba RJ, Liguori A, Marantz PR, Diane McKee M, Lypson ML. How to Make or Break Implicit Bias Instruction: Implications for Curriculum Development. Acad Med J Assoc Am Med Coll. 2018;93(11):S74-S81. doi:10.1097/ACM.0000000000002386

30. Mezirow J. Transformative Learning: Theory to Practice. New Dir Adult Contin Educ. 1997;1997(74):5-12. doi:https://doi.org/10.1002/ace.7401

31. Sukhera J, Watling CJ, Gonzalez CM. Implicit Bias in Health Professions: From Recognition to Transformation. Acad Med J Assoc Am Med Coll. 2020;95(5):717-723. doi:10.1097/ACM.0000000000003173

32. Ingraham N, Magrini D, Brooks J, Harbatkin D, Radix A, Haynes SG. Two Tailored Provider Curricula Promoting Healthy Weight in Lesbian and Bisexual Women. Womens Health Issues. 2016;26 Suppl 1:S36-42. doi:10.1016/j.whi.2016.04.001

33. Cahn Peter S. Recognizing and Reckoning With Unconscious Bias: A Workshop for Health Professions Faculty Search Committees. MedEdPORTAL. 13. doi:10.15766/mep_2374-8265.10544

34. Seeing Patients - Augustus A. White III, MD | Harvard University Press. Accessed January 12, 2021. https://www.hup.harvard.edu/catalog.php?isbn=9780674049055

35. Zeidan AJ, Khatri UG, Aysola J, et al. Implicit Bias Education and Emergency Medicine Training: Step One? Awareness. Aem Educ Train. 2019;3(1):81-85. doi:10.1002/aet2.10124

36. Neely KL, Stifel EN, Milberg LC. A systematic approach to faculty development in Women's health: lessons from education, feminism, and conflict theory. Acad Med. 2000;75(11):1095-1101.

37. Acosta D, Ackerman-Barger K. Breaking the Silence: Time to Talk About Race and Racism. Acad Med. 2017;92(3):285-288. doi:10.1097/ACM.0000000000001416

38. Adelekun AA, Beltrán S, Carney J, et al. Recognizing Racism in Medicine: A Student-Organized and Community-Engaged Health Professional Conference. Health Equity. 2019;3(1):395-402. doi:10.1089/heq.2019.0015

39. Holm AL, Rowe Gorosh M, Brady M, White-Perkins D. Recognizing Privilege and Bias: An Interactive Exercise to Expand Health Care Providers' Personal Awareness. Acad Med. 2017;92(3):360-364. doi:10.1097/ACM.0000000000001290 
40. Rodríguez JE, Campbell KM, Pololi LH. Addressing disparities in academic medicine: what of the minority tax? BMC Med Educ. 2015;15(1):6. doi:10.1186/s12909-015-0290-9

41. Like RC. Educating Clinicians About Cultural Competence and Disparities in Health and Health Care. J Contin Educ Health Prof. 2011;31(3):196-206. doi:10.1002/chp.20127

42. Frye AW, Hemmer PA. Program evaluation models and related theories: AMEE Guide No. 67. Med Teach. 2012;34(5):e288-e299. doi:10.3109/0142159X.2012.668637

43. Thomas PA, Kern DE, Hughes MT, Chen BY. Curriculum Development for Medical Education: A SixStep Approach. Johns Hopkins University Press; 2015. doi:10.1353/book.44600

44. Eva K, Regehr G. "I'll never play professional football" and other fallacies of self-assessment. J Contin Educ Health Prof. 2008;28:14-19. doi:10.1002/chp.150

45. Greenwald AG, Poehlman TA, Uhlmann EL, Banaji MR. Understanding and using the Implicit Association Test: III. Meta-analysis of predictive validity. J Pers Soc Psychol. 2009;97(1):17-41. doi:10.1037/a0015575

46. Olson MA, Fazio RH. Relations between implicit measures of prejudice:what are we measuring? Psychol Sci. 2003;14(6):636-639. doi:10.1046/j.0956-7976.2003.psci_1477.x

47. Olson MA, Fazio RH. Reducing the influence of extrapersonal associations on the Implicit Association Test: personalizing the IAT. J Pers Soc Psychol. 2004;86(5):653-667. doi:10.1037/00223514.86.5.653

48. Han HA, Czellar S, Olson MA, Fazio RH. Malleability of Attitudes or Malleability of the IAT? J Exp Soc Psychol. 2010;46(2):286-298. doi:10.1016/j.jesp.2009.11.011

49. Kirkpatrick DL. Seven keys to unlock the four levels of evaluation. Perform Improv. 2006;45(7):5-8. doi:https://doi.org/10.1002/pfi.2006.4930450702

50. Kennedy PE, Chyung SY, Winiecki DJ, Brinkerhoff RO. Training professionals' usage and understanding of Kirkpatrick's Level 3 and Level 4 evaluations. Int J Train Dev. 2014;18(1):1-21. doi:https://doi.org/10.1111/ijtd.12023

51. Richard P, Gary T. Evidence-Based Practice In Education. McGraw-Hill Education (UK); 2004.

52. Groccia JE, Buskist W. NEW DIRECTIONS FOR TEACHING AND LEARNING, no. 128, Winter 2011 () Wiley Periodicals, Inc. Published online in Wiley Online Library (wileyonlinelibrary.com) DOI: 10.1002/tl.463 1 Need for Evidence-Based Teaching.

\section{Figures}




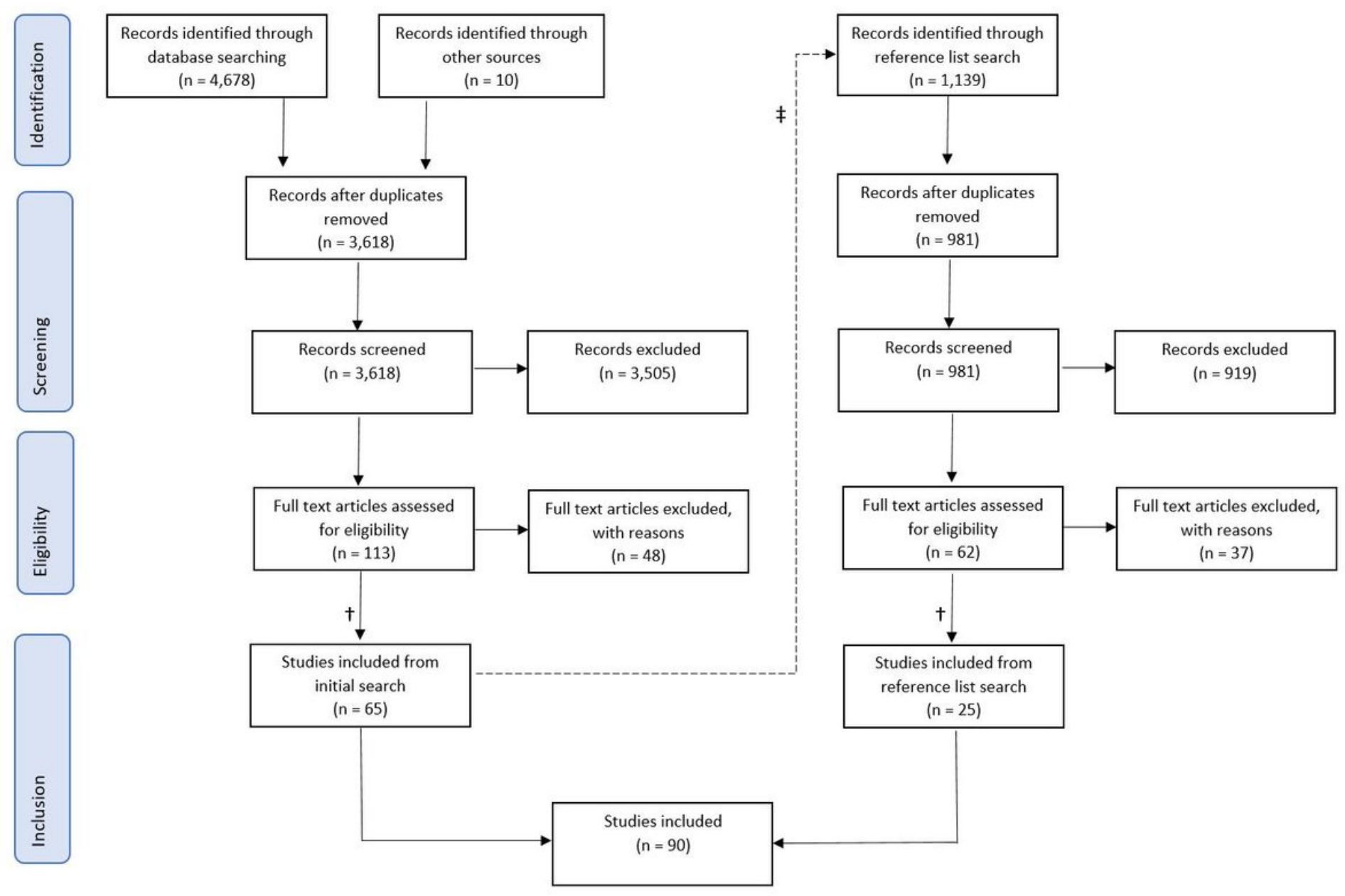

Figure 1

Flowchart of the screening process using PRISMA (Preferred Reporting Items for Systematic Reviews and Meta-Analyses) guidelines. ${ }^{11}$

† We assigned reviewers for full text review such that the screeners for each article's full text were different from the screeners for its title/abstract review. This ensured that each article was screened in total by 4 different reviewers, in order to minimize effects of individual biases or subjective interpretations of criteria.

¥ We imported sources cited in the bibliographies of included studies into Covidence and repeated the two-phase screening process. 


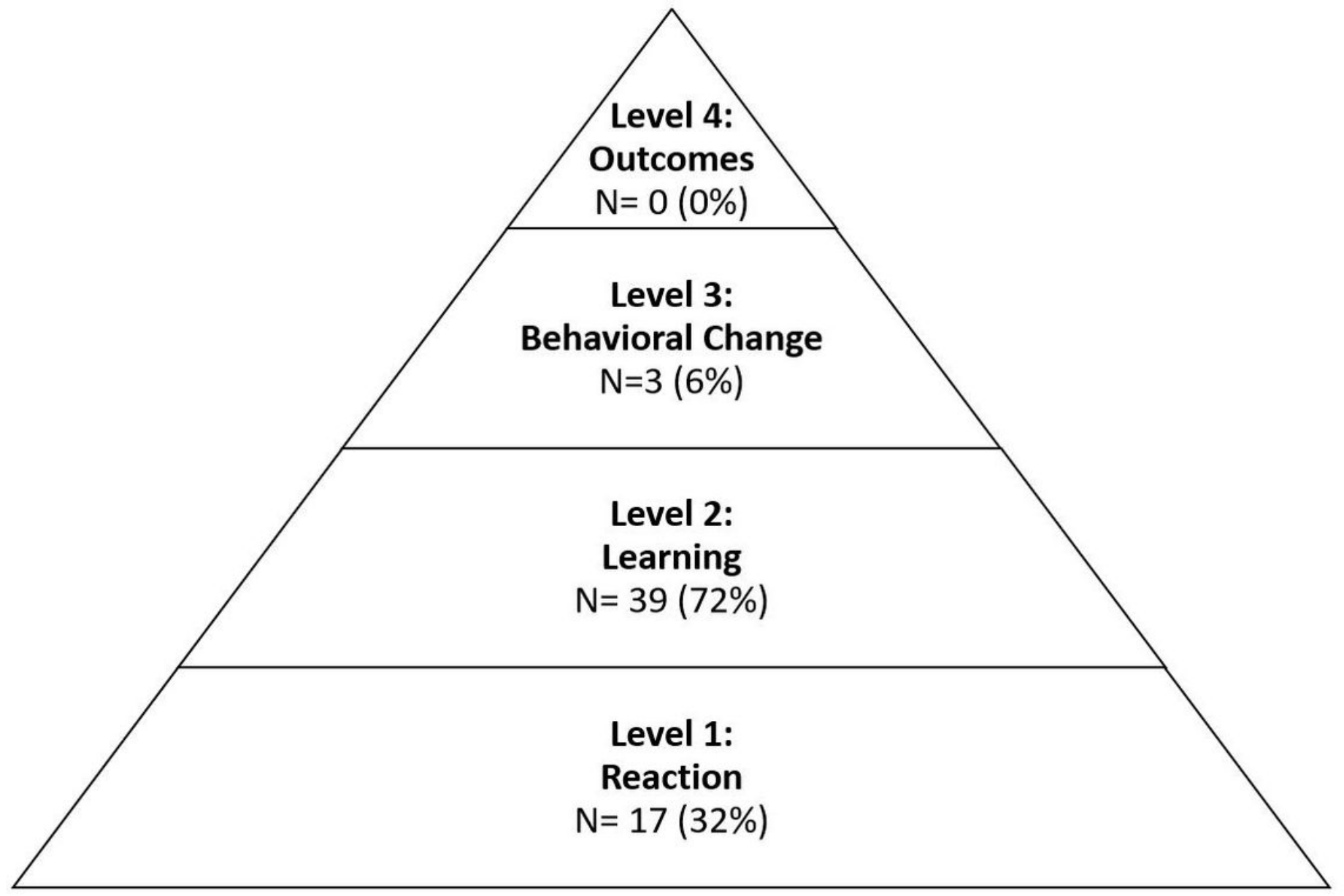

Figure 2

Number (\%) falling into each of the 4 levels of Kirkpatrick's Triangle for Program Evaluation, ${ }^{13}$ of 53 articles identified.

\section{Supplementary Files}

This is a list of supplementary files associated with this preprint. Click to download.

- Additionalfile1.docx

- Additionalfile2.docx

- Additionalfile3.docx 